\title{
Concentration at Half Tmax
}

National Cancer Institute

\section{Source}

National Cancer Institute. Concentration at Half Tmax. NCI Thesaurus. Code C135489.

The concentration that occurs at the midpoint time between dosing time and T max.

(CDISC) 